\title{
Synthesis of (E)-5-Arylvinyl-7-methyltetrazolo[1,5-a]pyrimidines
}

\author{
V. L. Gein ${ }^{a, *}$, A. N. Prudnikova ${ }^{a}$, A. A. Kurbatova ${ }^{a}$, and M. V. Dmitriev ${ }^{b}$ \\ ${ }^{a}$ Perm State Pharmaceutical Academy, Perm, 614990 Russia \\ ${ }^{b}$ Perm State National Research University, Perm, 614990 Russia \\ *e-mail: geinvl48@mail.ru
}

Received February 12, 2021; revised February 12, 2021; accepted February 24, 2021

\begin{abstract}
A three-component reaction of 5-aminotetrazole with aromatic aldehydes and acetylacetone under solvent- and catalyst-free conditions at a temperature of $150-160^{\circ} \mathrm{C}$ proceeds with the formation of $(E)-5$-arylvinyl7-methyltetrazolo[1,5-a]pyrimidines. 5,7-Dimethyltetrazolo[1,5-a]pyrimidine is formed as a side-product of the reaction.
\end{abstract}

Keywords: multicomponent reactions, 5-aminotetrazole, acetylacetone, tetrazolo[1,5- $a]$ pyrimidine

DOI: $10.1134 / \mathrm{S} 1070363221040071$

Multicomponent reactions are widely used for the synthesis of various organic compounds [1-5]. Tetrazolo[1,5-a]pyrimidine derivatives are promising and poorly studied heterocyclic compounds obtained by multicomponent reactions $[6,7]$. Compounds of this series have antimicrobial [8], antitumor [9], hypoglycemic [10], antiviral activities [11].

Earlier, various 7-aryl-substituted 4,7-dihydrotetrazolo[1,5-a]pyrimidine-6(5)-carboxylates were obtained using the three-component reaction of tetrazol-5-amine with aromatic aldehydes and 1,3-dicarbonyl compound, namely aroyl(hetaryl)pyruvic or acetoacetic acid esters $[12,13]$. The reaction was carried out by heating a mixture of starting reagents to $160^{\circ} \mathrm{C}$ in the absence of a solvent and a catalyst. Under these conditions, the reaction proceeded regioselectively to form the title compounds with high yields.

In continuation of the research, we performed the reaction using acetylacetone as a 1,3-dicarbonyl component. A mixture of $(E)$-5-arylvinyl-7-methyltetrazolo[1,5-a]pyrimidines $\mathbf{2 a}-\mathbf{2 e}$ and 5,7-dimethyltetrazolo[1,5-a]pyrimidine 3 was formed in a $6: 1$ ratio (according to ${ }^{1} \mathrm{H}$ NMR data) instead of the expected 7-aryl-6-acetyl-5methyl-4,7-dihydrotetrazolo[1,5-a]pyrimidines $\mathbf{1}$, when reacting equimolar amounts of acetylacetone, aromatic aldehyde and tetrazol-5-amine in solvent- and catalystfree conditions at $150-160^{\circ} \mathrm{C}$, (Scheme 1).
Compounds $\mathbf{2 a}-\mathbf{2 e}$ are yellow crystalline substances, poorly soluble in ethanol, dioxane, acetonitrile, readily soluble in acetic acid when heated, and insoluble in water and hexane.

The IR spectra of compounds $\mathbf{2 a}-\mathbf{2 e}$ and $\mathbf{3}$ contain absorption bands of medium intensity in the range of $1616-1624 \mathrm{~cm}^{-1}$, which are characteristic of stretching vibrations of the $\mathrm{C}=\mathrm{C}$ and $\mathrm{C}=\mathrm{N}$ bonds. The ${ }^{1} \mathrm{H}$ NMR spectra of compounds $\mathbf{2 a}-\mathbf{2 e}$ exhibit characteristic signals of the protons of the methyl group as a singlet at 2.91$2.94 \mathrm{ppm}$, the proton of the methine group of the heterocycle as a singlet at 7.69-7.75 ppm, and two olefinic protons as doublets at 7.30-7.57 and $8.02-8.28 \mathrm{ppm}$ $(J=16.0 \mathrm{~Hz})$, as well as protons of the aromatic ring and related groups.

The mass spectra of compounds $\mathbf{2 c}$ and $\mathbf{2 d}$ contain characteristic peaks of molecular ions with $\mathrm{m} / \mathrm{z} 304$ $[M-\mathrm{H}]^{-}$and $266[M-\mathrm{H}]^{-}$, respectively.

Compound $\mathbf{3}$ is a white crystalline substance, readily soluble in acetic acid, chloroform, acetone, poorly soluble in ethanol and insoluble in water. The ${ }^{1} \mathrm{H}$ NMR spectrum contains singlet signals of the protons of two methyl groups at 2.68 and $2.87 \mathrm{ppm}$, as well as multiplet signal of the methine proton at $7.36 \mathrm{ppm}$.

To confirm the proposed structure and establish the spatial structure of compounds $\mathbf{2 a - 2 e}$, attempts were made to obtain a single crystal by slow crystallization, 


\section{Scheme 1.}

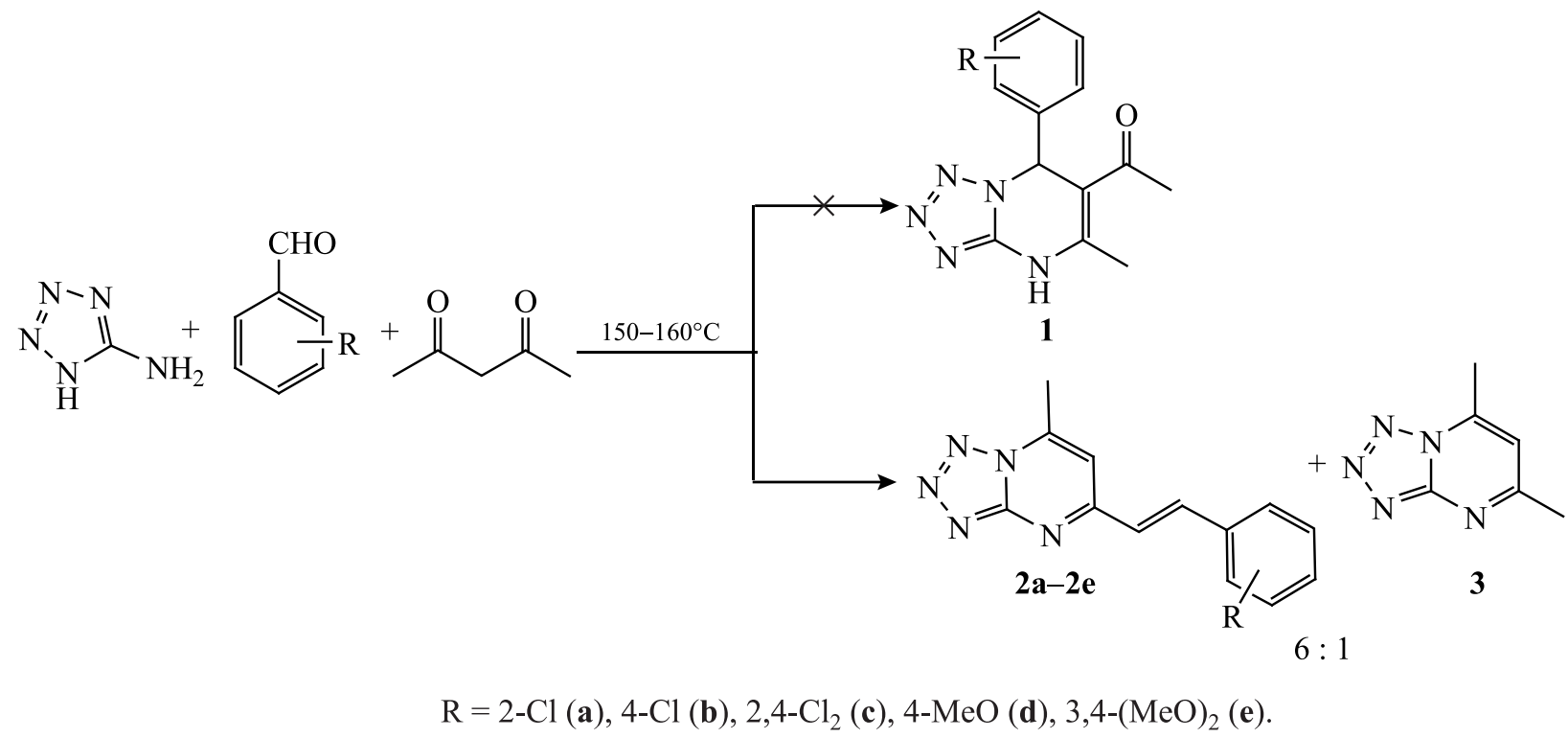

Scheme 2.
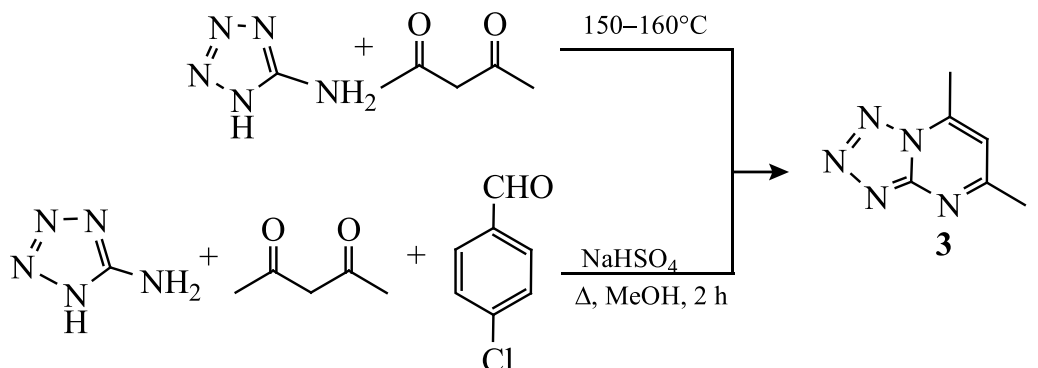

but crystals suitable for X-ray diffraction analysis were not obtained. Crystallization from acetic acid yielded a single crystal of compound 3 (Fig. 1).

Compound $\mathbf{3}$ crystallizes in the centrosymmetric space group of the monoclinic system. The bicyclic system of tetrazolopyrimidine is flat within $0.02 \AA$. The bond

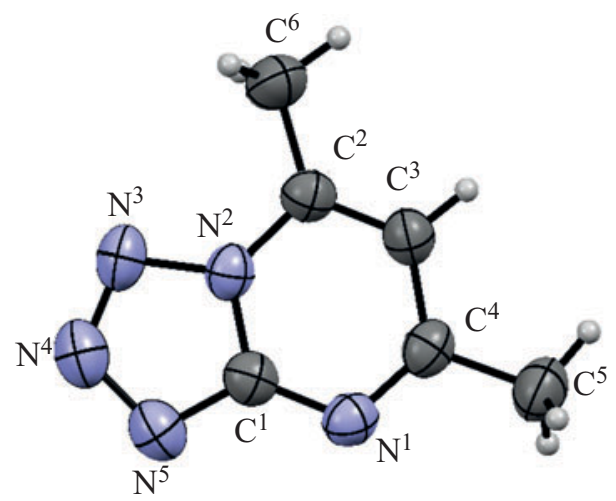

Fig. 1. General view of the molecule of compound 3 in the crystal. lengths and bond angles in the molecule have the typical values, with the exception of the slightly distorted $\mathrm{C}^{3} \mathrm{C}^{2} \mathrm{C}^{6}$ angle, $127.9(2)^{\circ}$. A similar deviation of the bond angle from $120^{\circ}$, leading to a shift of the methyl group $\mathrm{C}^{6} \mathrm{H}_{3}$ towards the tetrazole ring, is also typical for other alkylsubstituted tetrazolopyrimidines [14, 15].

Only 5,6-dimethyltetrazolo[1,5-a]pyrimidine 3 was obtained, when carrying out a three-component reaction under milder conditions in the presence of sodium hydrogen sulfate in methanol according to the previously described method [16]. Tetrazolo[1,5-a]pyrimidine 3 was also formed by direct fusion of 5-aminoterazole with acetylacetone (Scheme 2).

Probably, in the reaction between acetylacetone, aromatic aldehyde, and tetrazole-5amine, at the first stage, the addition of terazol-5-amine to acetylacetone occurs, followed by cyclization and the formation of intermediate $\mathbf{3}$ (Scheme 3 ). Compound $\mathbf{3}$ has a reactive methyl group, and when it reacts with an aromatic 
Scheme 3.

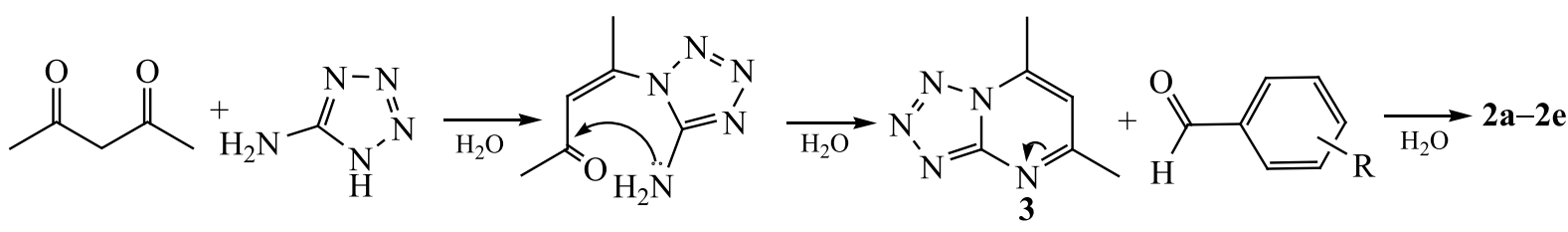

aldehyde, condensation occurs with the formation of (E)-5-(2-arylethenyl)-7-methyltetrazolo[1,5-a]pyrimidines $\mathbf{2 a}-\mathbf{2 e}$. The reaction stereoselectivity is due to the greater stability of the $E$-isomers in comparison with the $Z$-isomers. The low yield (5-22\%) of compounds 2 is probably associated with the occurrence of side reactions under these conditions.

In conclusion, the use of acetylacetone in a threecomponent reaction with a mixture of an aromatic aldehyde and tetrazol-5-amine leads to the formation of (E)-5-(2-arylethenyl)-7-methyltetrazolo[1,5-a]pyrimidines and 5,7-dimethyltetrazolo[1,5-a]pyrimidine.

\section{EXPERIMENTAL}

IR spectra were recorded on a FSM 1202 FT-IR spectrometer from mineral oil. ${ }^{1} \mathrm{H}$ NMR spectra were recorded on a Bruker AVANCE III HD 400 spectrometer from DMSO- $d_{6}$ solutions relative to internal TMS. Mass spectra were recorded on a Waters ACQUITY UPLC I-Class instrument by ultra-HPLC-MS method (Acquity UPLC BEH C18 $1.7 \mu \mathrm{m}$ column, acetonitrilewater mobile phases, flow rate $0.6 \mathrm{~mL} / \mathrm{min}$, Xevo TQD mass detector). Elemental analysis was performed on a PerkinElmer 2400 apparatus. Melting points were measured on a Melting Point M-565 instrument.

Single crystal X-ray diffraction analysis of compound 3 was performed on a Xcalibur Ruby diffractometer equipped with a CCD detector according to the standard method [Mo $K_{\alpha}$-radiation, 295(2) $\mathrm{K}$, $\omega$-scanning with a step of $1^{\circ}$ ]. Absorption was taken into account empirically using the SCALE3 ABSPACK algorithm [17]. The crystal system $\left(\mathrm{C}_{6} \mathrm{H}_{7} \mathrm{~N}_{5}, M 149.17\right)$ is monoclinic, space group $P 21 / c, a=8.064(3), b=12.456(6), c=7.074(3) \AA ; \beta=$ $91.03(4)^{\circ}, V=710.4(5) \AA^{3}, Z=4, d_{\text {calc }}=1.395 \mathrm{~g} / \mathrm{cm}^{3} ; \mu=$ $0.096 \mathrm{~mm}^{-1}$. The structure was solved using the SHELXS program [18] and refined by full-matrix least squares in $F^{2}$ in the anisotropic approximation for all non-hydrogen atoms using the SHELXL program [19] with the OLEX2 graphical interface [20]. When refining hydrogen atoms, the rider model was used. Final refinement parameters: $R_{1}=0.0550$ [for 1052 reflections with $I>2 \sigma(I)$ ], $w R_{2}=$
0.1766 (for all 1670 independent reflections), $S=1.026$. $\mathrm{X}$-Ray crystallographic parameters were deposited at the Cambridge Crystallographic Data Center (CCDC 2058640) and can be requested at www.ccdc.cam.ac.uk/ data_request/cif

(E)-5-[(2-Chlorophenyl)vinyl]-7-methyltetrazolo[1,5-a]pyrimidine (2a). A mixture of 0.01 mol $(1 \mathrm{~mL})$ of acetylacetone, $0.01 \mathrm{~mol}(1.1 \mathrm{~mL})$ of 2-chlorobenzaldehyde, $0.01 \mathrm{~mol}(1.03 \mathrm{~g})$ of tetrazol-5amine monohydrate was kept at $150-160^{\circ} \mathrm{C}$ until gas evolution ceased. The resulting mixture was cooled to room temperature, treated with ethanol. The precipitated crystals were filtered off and recrystallized from acetic acid. Yield $0.33 \mathrm{~g}(12 \%), \mathrm{mp} 192-194^{\circ} \mathrm{C}(\mathrm{AcOH})$. IR spectrum, $v, \mathrm{~cm}^{-1}: 2954\left(\mathrm{C}_{\mathrm{Alk}}-\mathrm{H}\right), 1616(\mathrm{C}=\mathrm{C}), 1462(\mathrm{Ar})$. ${ }^{1} \mathrm{H}$ NMR spectrum, $\delta$, ppm: $2.92 \mathrm{~s}\left(3 \mathrm{H}, \mathrm{CH}_{3}\right), 7.45-8.02$ $\mathrm{m}\left(4 \mathrm{H}_{\mathrm{Ar}}\right), 7.52 \mathrm{~d}\left(1 \mathrm{H}, \underline{\mathrm{CH}}_{\mathrm{A}}=\mathrm{CH}_{\mathrm{B}}, J=16.0 \mathrm{~Hz}\right), 7.72 \mathrm{~s}$ $\left(1 \mathrm{H}, \mathrm{C}^{6} \mathrm{H}\right) 8.28 \mathrm{~d}\left(1 \mathrm{H}, \mathrm{CH}_{\mathrm{A}}=\mathrm{CH}_{\mathrm{B}}, J=16.0 \mathrm{~Hz}\right)$. Found, \%: C 57.44; H 3.66; N 25.68. $\mathrm{C}_{13} \mathrm{H}_{10} \mathrm{ClN}_{5}$. Calculated, \%: C 57.42; H 3.68; N 25.76. M 256.04.

Compounds $\mathbf{2 b}-\mathbf{2} \mathbf{e}$ were prepared similarly.

(E)-5-[(4-Chlorophenyl)vinyl]-7-methyltetrazolo[1,5-a]pyrimidine (2b). Yield $0.42 \mathrm{~g}$ (15\%), mp 216$218^{\circ} \mathrm{C}(\mathrm{AcOH})$. IR spectrum, $v, \mathrm{~cm}^{-1}: 2932\left(\mathrm{C}_{\mathrm{Alk}}-\mathrm{H}\right)$, $1624(\mathrm{C}=\mathrm{C}), 1462$ (Ar). ${ }^{1} \mathrm{H}$ NMR spectrum, $\delta, \mathrm{ppm}: 2.93 \mathrm{~s}$ $\left(3 \mathrm{H}, \mathrm{CH}_{3}\right), 7.48 \mathrm{~d}\left(1 \mathrm{H}, \underline{\mathrm{CH}}_{\mathrm{A}}=\mathrm{CH}_{\mathrm{B}}, J=16.0 \mathrm{~Hz}\right), 7.55 \mathrm{~s}$ $\left(2 \mathrm{H}_{\mathrm{Ar}}\right), 7.84 \mathrm{~d}\left(2 \mathrm{H}_{\mathrm{Ar}}\right), 8.07 \mathrm{~s}\left(1 \mathrm{H}, \mathrm{CH}_{\mathrm{A}}=\mathrm{CH}_{\mathrm{B}}, J=16.0 \mathrm{~Hz}\right)$. Found, \%: C 57.51; H 3.62; N 25.74. $\mathrm{C}_{13} \mathrm{H}_{10} \mathrm{ClN}_{5}$. Calculated, \%: C 57.42; H 3.68; N 25.76. M 256.04

(E)-5-[(2,4-Dichlorophenyl)vinyl]-7-methyltetrazolo[1,5-a]pyrimidine (2c). Yield $0.67 \mathrm{~g}(22 \%), \mathrm{mp} 200$ $202^{\circ} \mathrm{C}(\mathrm{AcOH})$. IR spectrum, $v, \mathrm{~cm}^{-1}: 2924\left(\mathrm{C}_{\mathrm{Alk}}-\mathrm{H}\right)$, $1620(\mathrm{C}=\mathrm{C}), 1462(\mathrm{Ar}) .{ }^{1} \mathrm{H}$ NMR spectrum, $\delta$, ppm: $2.94 \mathrm{~s}$ $\left(3 \mathrm{H}, \mathrm{CH}_{3}\right), 7.57 \mathrm{~d}\left(1 \mathrm{H}, \underline{\mathrm{C}}_{\mathrm{A}}=\mathrm{CH}_{\mathrm{B}}, J=16.0 \mathrm{~Hz}\right), 7.58 \mathrm{~d}$ $\left(1 \mathrm{H}_{\mathrm{Ar}}, J=2.2 \mathrm{~Hz}\right), 7.77 \mathrm{~d}\left(1 \mathrm{H}_{\mathrm{Ar}}, J=2.2 \mathrm{~Hz}\right), 8.07 \mathrm{~d}\left(1 \mathrm{H}_{\mathrm{Ar}}\right.$, $J=8.4 \mathrm{~Hz}), 8.24 \mathrm{~d}\left(1 \mathrm{H}, \mathrm{CH}_{\mathrm{A}}=\mathrm{C}_{\mathrm{B}}, J=16.0 \mathrm{~Hz}\right)$. Mass spectrum, $m / z\left(I_{\text {rel }}, \%\right): 304(100)[M-\mathrm{H}]^{-}$. Found, \%: C 50.98; H 2.87; N 22.84. $\mathrm{C}_{13} \mathrm{H}_{9} \mathrm{Cl}_{2} \mathrm{~N}_{5}$. Calculated, \%: C 50.96; H 2.94; N 22.86. M 305.02. 
(E)-5-[(4-Methoxyphenyl)vinyl]-7-methyltetrazolo[1,5-a]pyrimidine (2d). Yield $0.15 \mathrm{~g}(6 \%), \mathrm{mp}$ 204-206 ${ }^{\circ} \mathrm{C}(\mathrm{AcOH})$. IR spectrum, $v, \mathrm{~cm}^{-1}: 2916\left(\mathrm{C}_{\mathrm{Alk}}-\mathrm{H}\right)$, $1624(\mathrm{C}=\mathrm{C}), 1462(\mathrm{Ar}) .{ }^{1} \mathrm{H}$ NMR spectrum, $\delta$, ppm: $2.91 \mathrm{~s}$ $\left(3 \mathrm{H}, \mathrm{CH}_{3}\right), 3.81 \mathrm{~s}\left(3 \mathrm{H}, \mathrm{CH}_{3} \mathrm{O}\right), 7.05-7.76 \mathrm{~m}\left(4 \mathrm{H}_{\mathrm{Ar}}\right), 7.30 \mathrm{~d}$ $\left(1 \mathrm{H}, \underline{\mathrm{C}}_{\mathrm{A}}=\mathrm{CH}_{\mathrm{B}}, J=16.0 \mathrm{~Hz}\right), 8.04 \mathrm{~d}\left(1 \mathrm{H}, \mathrm{CH}_{\mathrm{A}}=\underline{\mathrm{C}}_{\mathrm{B}}\right.$, $J=16.0 \mathrm{~Hz})$. Mass spectrum, $m / z\left(I_{\text {rel }}, \%\right): 266(95)$ $[M-\mathrm{H}]^{-}$. Found, \%: C 62.96; H 4.79; N 26.28. $\mathrm{C}_{14} \mathrm{H}_{13} \mathrm{~N}_{5} \mathrm{O}$. Calculated, \%: C 62.85; H 4.86; N 26.29. M 267.11

(E)-5-[(3,4-Dimethoxyphenyl)vinyl]-7-methyltetrazolo[1,5-a]pyrimidine (2e). Yield $0.13 \mathrm{~g} \mathrm{(5 \% ),} \mathrm{mp} \mathrm{198-}$ $200^{\circ} \mathrm{C}(\mathrm{AcOH})$. IR spectrum, $v, \mathrm{~cm}^{-1}: 2912\left(\mathrm{C}_{\mathrm{Alk}}-\mathrm{H}\right)$, $1612(\mathrm{C}=\mathrm{C}), 1377$ (Ar). ${ }^{1} \mathrm{H}$ NMR spectrum, $\delta$, ppm: $2.91 \mathrm{~s}$ $\left(3 \mathrm{H}, \mathrm{CH}_{3}\right), 3.84 \mathrm{c}\left(3 \mathrm{H}, \mathrm{CH}_{3} \mathrm{O}\right), 3.87 \mathrm{~s}\left(3 \mathrm{H}, \mathrm{CH}_{3} \mathrm{O}\right)$, $7.06-7.44 \mathrm{~m}\left(3 \mathrm{H}_{\mathrm{Ar}}\right), 7.36 \mathrm{~d}\left(1 \mathrm{H}, \underline{\mathrm{CH}}_{\mathrm{A}}=\mathrm{CH}_{\mathrm{B}}, J=16.0 \mathrm{~Hz}\right)$, $8.02 \mathrm{~d}\left(1 \mathrm{H}, \mathrm{CH}_{\mathrm{A}}=\mathrm{C}_{\mathrm{B}}, J=16.0 \mathrm{~Hz}\right)$. Found, \%: C 60.48; $\mathrm{H}$ 5.64; N 23.51. $\mathrm{C}_{15} \mathrm{H}_{15} \mathrm{~N}_{5} \mathrm{O}_{2}$. Calculated, \%: C 60.54; H 5.61; N 23.54. M 297.12

5,7-Dimethyltetrazolo[1,5-a]pyrimidine (3). $a$. A mixture of $0.01 \mathrm{~mol}(1 \mathrm{~mL})$ of acetylacetone, $0.01 \mathrm{~mol}$ $(1.03 \mathrm{~g})$ of tetrazol-5-amine monohydrate was kept for 2 min at $150-160^{\circ} \mathrm{C}$ until the reaction mixture solidified. The residue was cooled to room temperature and treated with ethanol. The precipitated crystals were filtered off and recrystallized from ethanol. Yield 80\%.

$b$. To a solution of $0.01 \mathrm{~mol}(1 \mathrm{~mL})$ of acetylacetone, $0.01 \mathrm{~mol}(1.1 \mathrm{~mL})$ of 2-chlorobenzaldehyde, $0.01 \mathrm{~mol}$ $(1.03 \mathrm{~g})$ of tetrazol-5-amine monohydrate in $15 \mathrm{~mL}$ of methanol was added $0.001 \mathrm{~mol}(0.12 \mathrm{~g})$ of $\mathrm{NaHSO}_{4}$. The resulting mixture was boiled for $2 \mathrm{~h}$. The precipitated crystals were filtered off, washed with water, dried at room temperature, and recrystallized from ethanol. Yield $77 \%, \mathrm{mp} 150-152^{\circ} \mathrm{C}$ (EtOH). IR spectrum, $v, \mathrm{~cm}^{-1}: 1624$ $(\mathrm{C}=\mathrm{C}), 1531(\mathrm{C}=\mathrm{N}), 1377\left(\mathrm{C}-\mathrm{CH}_{3}\right) .{ }^{1} \mathrm{HNMR}$ spectrum, $\delta$, ppm: $2.68 \mathrm{~s}$ and $2.87 \mathrm{~s}\left(6 \mathrm{H}, \mathrm{CH}_{3}\right), 7.36 \mathrm{~m}(1 \mathrm{H}, \mathrm{CH})$. Found, \%: $\mathrm{C} 48.25 ; \mathrm{H} 4.71 ; \mathrm{N} 46.89 . \mathrm{C}_{6} \mathrm{H}_{7} \mathrm{~N}_{5}$. Calculated, \%: C 48.27; H 4.69; N 46.93. M 149.07.

\section{CONFLICT OF INTEREST}

No conflict of interest was declared by the authors.

\section{OPEN ACCESS}

This article is licensed under a Creative Commons Attribution 4.0 International License, which permits use, sharing, adaptation, distribution and reproduction in any medium or format, as long as you give appropriate credit to the original author(s) and the source, provide a link to the Creative
Commons licence, and indicate if changes were made. The images or other third party material in this article are included in the article's Creative Commons licence, unless indicated otherwise in a credit line to the material. If material is not included in the article's Creative Commons licence and your intended use is not permitted by statutory regulation or exceeds the permitted use, you will need to obtain permission directly from the copyright holder. To view a copy of this licence, visit http://creativecommons.org/licenses/by/4.0/.

\section{REFERENCES}

1. Mironov, M.A., Ros. Khim. Zh., 2009, vol. 53, no. 5, p. 116.

2. Ruijter, E. and Orru, R.V.A., Drug Discov Today Technol., 2013, vol. 10, p. e15. https://doi.org/10.1016/j.ddtec.2012.10.012

3. Cioc, R.C., Ruijter, E., and Orru, R.V.A., Green Chem., 2014, vol. 16, p. 2958. https://doi.org/10.1039/C4GC00013G

4. Malinakova, H.C., Rep. Org. Chem., 2015, vol. 5, p. 75. https://doi.org/10.2147/ROC.S65115

5. Heravi, M.M., Zadsirjan, V., Dehghani, M., and Ahmadi, T., Tetrahedron, 2018, vol. 74, p. 3391. https://doi.org/10.1016/j.tet.2018.04.076

6. Neochoritis, C.G., Zhao, T., and Dömling, A., Chem. Rev., 2019, vol. 119, p. 1970.

https://doi.org/10.1021/acs.chemrev.8b00564

7. Dolzhenko, A.V., Heterocycles, 2017, vol. 94, no. 10, p. 1819. https://doi.org/10.3987/REV-17-867

8. Scapin, E., Frizzo, C.P., Rodrigues, L.V., Zimmer, G.C., Vaucher, R.A., Sagrillo, M.R., Giongo, J.L., Afonso, C.A.M., Rijo, P., and Zanatta, N.B., Med. Chem. Res., 2017, vol. 26, p. 640. https://doi.org/10.1007/s00044-017-1783-3

9. Dougherty, A., Guo, H., Westby, G., Liu, Y., Simsek, E., Guo, J., Mehta, A., Norton, P., Gu, B., Block, T., and Cuconati, A., Antimicrob. Agents Chemother, 2007, vol. 51, p. 4427. https://doi.org/10.1128/AAC.00541-07

10. Popova, E.A., Protas, A.V., and Trifonov, R.E., Anticancer Agents Med. Chem., 2017, vol. 17, p. 1856. https://doi.org/10.2174/1871520617666170327143148

11. Gein, V.L., Zamaraeva, T.M., Mishunin, V.V., and Kotegov, V.P., Russ. J. Gen. Chem., 2016, vol. 86, no. 2, p. 286. https://doi.org/10.1134/S1070363216020134

12. Gein, V.L., Gein, L.F., Tsyplyakova, E.P., and Rozova, E.A., Russ. J. Org. Chem., 2003, vol. 39, no. 5, p. 753. https://doi.org/10.1023/A:1026002522354 
13. Gein, V.L. and Zamaraeva, T.M., Russ. J. Gen. Chem., vol. 86, no. 1, p. 196.

https://doi.org/10.1134/S1070363216010321

14. Russ, T., Bats, J.W., and Ried, W., CSD Commun. (Private Communication), 1992. CSD 54666.

15. Scapin, E., Salbego, P.R.S., Bender, C.R., Meyer, A.R., Pagliari, A.B., Orlando, T., Zimmer, G.C., Frizzo, C.P., Bonacorso, H.G., Zanatta, N., and Martins, M.A.P., Beilstein J. Org. Chem., 2017, vol. 13, p. 2396.

https://doi.org/10.3762/bjoc.13.237

16. Gein, V.L., Gorgopina, E.V., Zamaraeva, T.M., and Dmitriev, M.V., Russ. J. Org. Chem., 2017, vol. 53, no. 11, p. 1675.

https://doi.org/10.1134/S1070428017110100

17. CrysAlisPro, Agilent Technologies, Version 1.171.37.33.

18. Sheldrick, G.M., Acta Crystallogr. (A), 2008, vol. 64, p. 112. https://doi.org/10.1107/S0108767307043930

19. Sheldrick, G.M., Acta Crystallogr. (C), 2015, vol. 71, p. 3.

https://doi.org/10.1107/S2053229614024218

20. Dolomanov, O.V., Bourhis, L.J., Gildea, R.J., Howard, J.A.K., and Puschmann, H., J. Appl. Cryst., 2009, vol. 42 , p. 339.

https://doi.org/10.1107/S0021889808042726 